\title{
Manía inducida por el tratamiento con corticoesteroides: revisión a partir de un caso clínico.
}

Corticosteroid-induced mania: a case study and review.

\author{
María Dolores Sánchez García a , Beatriz Pecino Esquerdo b, Enrique Pérez Martínez c . \\ ${ }^{a, b y c}$ Servicio de Psiquiatría. ${ }^{a}$ Complejo Hospitalario Universitario de Albacete, España ${ }^{b}$ Hospital Clínico \\ de San Juan de Alicante, España ${ }^{c}$ Hospital General Universitario de Alicante, España. \\ Correspondencia: Enrique Pérez Martínez (eperezmar@yahoo.es)
}

Recibido: 29/04/2014; aceptado con modificaciones: 28/12/2014

\begin{abstract}
RESUMEN: Los corticoides son fármacos muy usados en la práctica clínica habitual que, sin embargo, se asocian a frecuentes efectos adversos neuropsiquiátricos. Se describe el caso clínico de un varón de setenta y un años que desarrolló síntomas maníacos tras toma de corticoides y que fue tratado de forma satisfactoria con quetiapina. A partir de este caso, se realiza una revisión de la manía inducida por corticoides.

PALABRAS CLAVE: trastorno bipolar, episodio maníaco, corticoides, oncología médica, quetiapina.
\end{abstract}

\begin{abstract}
Corticosteroids are drugs widely used in routine clinical practice that are however associated with neuropsychiatric adverse effects. This paper describes the case of a seventy-one- year man who developed manic symptoms after taking with corticosteroids and was successfully treated with quetiapine and, on the other hand, reviews corticosteroid-induced mania.

KEY WORDS: bipolar disorder, mania, adrenal cortex hormones, medical oncology, quetiapine.
\end{abstract}

\section{Introducción.}

La prescripción de los glucocorticoides (en adelante se utilizará de forma indistinta glucocorticoide, corticoide y corticosteroide), como la prednisona o la metilprednisolona, se lleva realizando desde hace sesenta años. Sus propiedades antiinflamatorias e inmunosupresoras los han convertido en un tratamiento muy extendido y altamente efectivo para un importante número de patologías médicas, que incluyen los trastornos inmunológicos e inflamatorios, el lupus eritematoso sistémico, las vasculitis sistémicas, el asma y las enfermedades pulmonares obstructivas crónicas, el cáncer, el dolor agudo y crónico de espalda, la prevención de la inflamación tras cirugía de cuello y cabeza así como para evitar el rechazo post-transplante (1). Pero a pesar de todas estas propiedades beneficiosas, también están asociados a múltiples efectos no deseados como la pérdida ósea, glaucoma, cataratas, infecciones, diabetes y problemas psiquiátricos en un cierto número de pacientes. Las primeras reacciones psiquiátricas inducidas por corticoides fueron descritas a principio de la década de los años 50 del siglo pasado y se denominaron como "psicosis esteroideas", ya que presentaban el aspecto de una bouffée delirante aguda con síntomas disfóricos (2-3). Posteriormente empezaron a aparecer en la literatura casos de depresión, manía y delirium. Aunque el dato más sorprendente encontrado fue el incremento del riesgo de suicidio y de intento de suicidio en seis veces, especialmente en la gente más joven (4). 
Tabla 1:

Síntomas neuropsiquiátricos originados por la corticoterapia.

\begin{tabular}{|l|}
\hline Síntomas neuropsiquiátricos originados por la corticoterapia \\
\hline -Depresión. \\
-Hipomanía. \\
-Manía. \\
- Psicosis. \\
-Ideas de suicidio. \\
-Irritabilidad. \\
-Agresividad. \\
-Ansiedad. \\
-Trastornos del sueño. \\
\hline
\end{tabular}

Cambios emocionales: Son difíciles de evaluar pero a menudo están presentes previamente al inicio del tratamiento y pueden estar relacionados con la disminución en la calidad de vida que origina la enfermedad concomitante.

Crisis de agresividad: Se ha observado que en niños son más frecuentes y aparecen tempranas alteraciones comportamentales.

Depresión: La sintomatología depresiva se incrementa durante la terapia.

Crisis de agitación o manía: Es frecuente observar que los primeros días los pacientes presentan síntomas de agitación y que pueden estar dentro del contexto de episodios hipomaníacos o maníacos.

Ideas de suicidio: Se ha apuntado a que también eran dosis-dependientes $\mathrm{y}$ que no mostraban relación con la severidad de la patología médica concomitante ni con antecedentes psiquiátricos previos.

Trastornos de la memoria: Existen compromisos de la memoria verbal y de la memoria a corto plazo (5).

Se calcula que la incidencia de complicaciones psiquiátricas secundarias a corticoides oscila entre un $6 \%$ de reacciones severas, un $28 \%$ de reacciones moderadas, y puede alcanzar hasta el $72 \%$ si incluimos las reacciones leves (5- 8). La asociación entre los corticoides exógenos y los síntomas afectivos está bien establecida y en estudios retrospectivos encontramos tasas que oscilan entre el 1 y el $50 \%(9)$.

El DSM-IV incluye los siguientes trastornos inducidos por corticoides: trastornos del ánimo (manía, hipomanía, depresión y trastornos mixtos), trastornos de ansiedad, psicosis y delirium. Los cuadros más frecuentes son depresión (28-41\%) y manía (29-35\%), seguidos de psicosis (11-14\%), delirium (10-13\%) y estados mixtos (8-12\%) (10-12). Se ha descrito también la existencia de un cuadro de demencia reversible inducido por corticoides (1), así como una amplia variedad de síntomas como disforia, síntomas de abstinencia, síntomas del espectro obsesivocompulsivo, heteroagresividad, catatonia y mal uso/abuso de corticoides (7). En 2012 se publicó un estudio (9) cuyo objetivo era describir las tasas de incidencia 
de depresión, manía, delirium, trastorno de pánico y suicidio consumado / gestos suicidas en pacientes tratados con corticoides en comparación con un grupo de pacientes sin tratamiento. Comparado con población no expuesta, el riesgo de trastorno depresivo aumenta dos veces; de cuatro a cinco veces el de manía o cuadros confusionales, y el de suicidio o gestos suicidas aumenta de cinco a siete veces en el grupo de pacientes en tratamiento corticoideo.

Hemos de tener en cuenta que estos datos pueden estar infraestimados ya que con frecuencia los clínicos no reportan todos los efectos adversos que aparecen durante el tratamiento.

A pesar de que es bien conocido el riesgo de desarrollar síntomas psiquiátricos durante el tratamiento corticoideo, la fisiopatología de estas reacciones adversas no es bien conocida en la actualidad, pero clínicamente es importante conocer su existencia así como los posibles métodos de prevención y tratamiento de las mismas.

En el caso de los pacientes oncológicos, e independientemente del uso de corticoides, encontramos que aproximadamente la mitad presentan sintomatología psiquiátrica, siendo los trastornos adaptativos, la depresión y el delirium los trastornos más prevalentes, mientras que los cuadros maniformes son mucho menos frecuentes (13).

Pese a que la evidencia es escasa, algunas investigaciones apuntan a que los corticoides podrían tener un papel en el comienzo del trastorno bipolar tipo I primario (12).

\section{Sintomas y sindromes maníacos inducidos por corticoesteroides.}

Los síntomas y síndromes maníacos inducidos por corticoesteroides se clasifican como "Trastorno maníaco orgánico" en la CIE-10 (14) y como "Trastorno del estado de ánimo inducido por sustancias, con síntomas maníacos" en el DSM-IVTR (15).

Hasta el 75\% de los pacientes tratados con corticoides pueden desarrollar síntomas psiquiátricos leves, mientras que los problemas graves oscilan entre el $5-18 \%$ de los casos $(3,5,12,16)$. El riesgo aumenta a partir de una dosis diaria de $40 \mathrm{mg} /$ día, pasando de un 1,3\% de reacciones psiquiátricas en dosis inferiores a 40 $\mathrm{mg} /$ día, a un 4,6\% en dosis de $40 \mathrm{mg} /$ día y alcanzando el $18,4 \%$ en dosis superiores (17).

Los trastornos del humor son los problemas más frecuentes y documentados en la literatura, algunos estudios señalan que son los cuadros de características hipomaníacas y maníacas los predominantes en la toxicidad psiquiátrica por corti- 
coides $(1,12,18)$. Los síntomas psicóticos se observan con frecuencia en el curso de los episodios maníacos. Solamente en un estudio de pacientes oncológicos tratados con altas dosis de corticosteroides, los autores encontraron una predominancia de aparición de cuadros de delirium en lugar de síndromes afectivos. Esto podría deberse a las complicaciones médicas de éstos pacientes, el frecuente uso de narcóticos y a los propios efectos neurológicos del cáncer (19).

En los casos de manía/hipomanía, la dosis media de dosis equivalente de prednisona fue de $44.8 \pm 45.6 \mathrm{mg} /$ día y el tiempo necesario para la recuperación de $21 \pm 18.8$ días. Sugieren además que los síntomas maníacos aparecen con dosis menores de corticoide respecto al delirium (1).

La duración de la sintomatología se encuentra entre 2 y 60 días con una media de 21 días, no habiéndose encontrado diferencias significativas entre cuadros depresivos, maníacos o psicóticos, salvo en el delirium que es el de menor duración. La recuperación es completa en el $93 \%$ de los casos, el $4 \%$ continúa con sintomatología o presenta ésta de forma recurrente y el 3\% se suicida (20).

\section{Incidencia de cuadros maníacos.}

Ya en 1952 Rome and Braceland publicaron tasas de incidencia de efectos secundarios por corticoides en los que un $60 \%$ de casos desarrollaban cuadros de euforia o manía (3). En una revisión de 139 tratamientos en una muestra de 135 pacientes con LES, sin síntomas de afectación neurológica, tratados con corticoides aparecieron nueve casos de novo de episodios maníacos (6,5\%) (21).

En el estudio clásico de Lewis y Smith (11) sobre 79 casos con alteraciones psiquiátricas encontraron que 22 correspondían a cuadros de manía $(28 \%)$ y 6 a cuadros mixtos (7\%). En la revisión de Sirois sobre publicaciones de casos de psicosis esteroideas entre 1970 y 1983 (22), se describe la aparición de un 35\% de casos de manía y un $12 \%$ de manía y depresión.

En un servicio psiquiátrico hospitalario los corticoides causaron el $54 \%$ de las manías orgánicas. En un estudio prospectivo de 50 pacientes, 13 de ellos desarrollaron hipomanía (26\%) (12). Otro estudio prospectivo con 50 pacientes que ingresaron en una unidad de medicina interna y que recibieron tratamiento con corticoides, encontró 14 casos de hipomanía (28\%) administrando la escala de hipomanía de Angst (3).

Clásicamente se aceptaba que era en los tratamientos breves con corticoides donde la incidencia de cuadros maníacos era mayor mientras que en los tratamientos a largo plazo se describían síntomas depresivos con mayor frecuencia (23-24). Sin embargo estudios recientes utilizando escalas autoadministradas como la Internal 
ORIGINALES Y REVISIONES

State Scale (ISS), que es más sensible a los efectos de los corticoides sobre el humor, detectan que hasta el $60 \%$ de sujetos tratados a largo plazo cumplían criterios de hipomanía/manía (que las escalas clínicas como la $Y M R S$ no detectaban) (23).

\section{Debut de la sintomatología maníaca.}

Los síntomas maníacos pueden aparecer en un período que va desde menos de 24 horas del inicio del tratamiento corticoideo hasta varios meses después de iniciado el tratamiento. Dicho período no parece correlacionar con las características del tratamiento corticoideo ni con la enfermedad somática de base (25).

Los estudios clásicos establecen su aparición entre los tres y cuatro días, con una media de once días tras iniciar el tratamiento (12-13), pero pueden aparecer hasta los 54 días. La mayor parte de los pacientes los desarrollan durante la primera semana (43\%), el $57 \%$ en las dos primeras semanas y el $93 \%$ dentro de las seis primeras (11).

Naber et al presentaron un estudio prospectivo de una cohorte de 50 pacientes que recibían tratamiento con metilprednisona o fluocortolona con dosis iniciales de $119 \pm 41 \mathrm{mg} /$ día y $75 \pm 22 \mathrm{mg}$ /día durante más de ocho días, encontrando síntomas maníacos en un $26 \%$ de los participantes, la mayoría de ellos comenzaron en los tres primeros días del tratamiento y continuaron, a pesar de la disminución, hasta los 8 días del estudio (26).

En un estudio prospectivo con 50 pacientes que tomaron corticoides durante un período de seis meses a cinco años, los síntomas aparecieron en el primer mes de tratamiento (3) y eran vividos positivamente por los pacientes que los interpretaban como un signo de mejoría.

La dexametasona y la betametasona tienen vidas medias de 36-54 horas por lo que pueden acumularse e inducir la aparición de síntomas psiquiátricos con posterioridad a la última dosis administrada (1).

Tras la discontinuación de la terapia esteroidea los síntomas de manía pueden persistir durante tres semanas (12-13).

Se ha apuntado a que los síntomas neuropsiquiátricos que se observan durante la terapia corticoidea simulan un trastorno bipolar, ya que pueden comenzar con síntomas depresivos y compromiso de la memoria y días más tarde aparece un cuadro de agitación maníaca franca. No hay que olvidar que además de estos síntomas, se encuentran los físicos como son el aumento de peso, facies de luna llena, ardor epigástrico, enrojecimiento facial y/o síndrome de Cushing. 


\section{Correlación entre dosis de corticoesteroides y aparición de síntomas maniacos.}

Revisando la literatura, la mayoría de los estudios coinciden en afirmar que el riesgo de desarrollar síntomas psiquiátricos inducidos por los corticoides presenta una relación dosis-respuesta, especialmente con dosis a partir de 30-40 mg/día o 1 $\mathrm{mg} / \mathrm{kg} / \mathrm{dia}$ de prednisona o equivalente $(1,3,16)$, no encontrándose relación con el tiempo, la severidad o la duración del efecto $(1,6,8,27)$ y concluyéndose que la dosis diaria inicial de corticoides puede ser predictiva de los efectos adversos psiquiátricos (9). Nishimura et al publicaron que con dosis mayores de 40mg de prednisolona/día se incrementaba el riesgo de desarrollar clínica. Además si hablamos de dosis altas, en este caso de metilprednisolona $(500-100 \mathrm{mg}$ ), se incrementa la probabilidad de hipomanía/manía (7). Si se trata de dexametasona, según Kenna et al, la dosis media con la que aparecen los síntomas es de $9.1 \mathrm{mg} /$ día $(7,13)$.

Como se ha apuntado anteriormente, los síntomas suelen aparecer en las primeras semanas de tratamiento y generalmente revierten tras la interrupción del mismo (6). Sin embargo, es importante señalar que al suspender la terapia corticoidea también pueden presentarse alteraciones del afecto y de la cognición, parecidos a los que se observan tras el inicio de ésta, describiéndose cuadros de confusión mental, agitación e incluso clínica alucinatorio-delirante en pacientes con tratamiento crónico a los que se les ha suspendido la medicación. En este caso, los síntomas mejoran al reintroducir el corticoide, o uno similar a dosis bajas. En este momento no existe una explicación clara para este hecho clínico (5).

\section{Otros factores de riesgo.}

Mientras que la dosis es un factor de riesgo conocido, el papel de otros factores como la edad, el sexo y antecedentes de patología médica, no han podido considerarse consistentes $(6,9)$. Los antecedentes psiquiátricos en general no se consideran un factor de riesgo por la mayoría de autores $(1,25,27)$, aunque algún estudio si que ha encontrado relación entre patología psiquiátrica previa y desarrollo de secundarismos (9).

Especialmente analizado ha sido el género, ya que algunos autores hacen referencia a un mayor riesgo en mujeres $(1,27)$. Este hallazgo podría explicarse en el contexto de que muchas enfermedades que precisan tratamiento con corticoides son más frecuentes en ellas, como por ejemplo el lupus eritematoso sistémico o la artritis reumatoide, pudiendo ser el sexo un factor de confusión. Además el sexo femenino suele desarrollar depresión y en el caso de ellos, manía o delirium.

Por último, también se ha visto que el riesgo de reacciones adversas aumenta en 
ciertas situaciones clínicas: utilización de fármacos que elevan los niveles circulantes de corticosteroides (como es el caso de la claritromicina que inhibe el citocromo P450), en aquellos pacientes en cuyo LCR está disminuida la tasa de albúmina (marcador de daño en la barrera hematoencefálica) y en casos de hipoalbuminemia e hipoalbuminuria (1).

Si consideramos aquellos pacientes que reciben múltiples tandas de corticoides, una historia previa de trastornos psiquiátricos inducidos se asocia con mayor riesgo de recurrencia del secundarismo, aproximadamente un aumento del $32 \%$ según datos obtenidos (4). Por eso, aunque algunos autores proponen que un único trastorno psiquiátrico secundario a corticoides no debe ser una contraindicación para tratamientos corticoideos futuros (9), otros apoyan la contraindicación de terapia corticoidea en pacientes con historia previa de psicosis (11).

Por otro lado, los pacientes que no experimentaron efectos secundarios por corticoides en el pasado no parecen estar protegidos si vuelven a necesitar un tratamiento con esteroides, pues un estudio de 17 casos de trastornos psiquiátricos inducidos por corticoides en pacientes que tenían antecedentes de tratamientos previos, encontró que seis de ellos tuvieron problemas psiquiátricos previos cuando tomaron corticoides y once de ellos no (12-13).

\section{Fisiopatología.}

La fisiopatología subyacente a los efectos neuropsiquiátricos de los corticoides no ha sido bien definida, habiéndose propuesto diferentes mecanismos. Parece que los corticoides actúan sobre distintos receptores específicos, siendo los de tipo II, que predominan en el cerebro anterior, los que parecen modular la respuesta emocional. Así, una sobreexpresión de estos receptores podría estar en la base de la fisiopatología del trastorno maníaco. Otra explicación es el efecto en los sistemas dopaminérgico, colinérgico y serotoninérgico, donde estaría implicada la hiperactividad de la vía dopaminérgica y la disminución en la liberación de serotonina. En los últimos años se está prestando especial atención al papel del sistema inmune, sobre todo en relación con los niveles de factor alfa de necrosis tumoral (TNF- $\alpha$ ), IL-1, IL-6 y factor de TNF soluble de los receptores, aunque de momento los resultados son preliminares $(1,10,13)$. Especialmente importantes parecen los estudios sobre el efecto de los corticoides en el hipocampo, siendo ésta la estructura diana de la actividad cerebral de los corticoides y que parece ser la responsable de los cambios en el afecto, pensamiento y comportamiento de los pacientes. Los corticoides producirían una atrofia y disminución del volumen hipocampal, cambios descritos en enfermedades como los trastornos afectivos, la enfermedad de Alzhei- 
mer y el trastorno por estrés postraumático (20). En primates y roedores se ha visto que el exceso de corticoides produce atrofia de las dendritas, astrogliosis y pérdida de neuronas hipocampales. En humanos no se han obtenido los mismos resultados aunque se han señalado cambios en la organización de las sinapsis y patrones de astrogliosis reactiva de algunas áreas $(5,20)$. El hipocampo está implicado en la consolidación de la memoria a corto plazo y déficits en ésta se han descrito en sujetos expuestos a altas dosis de corticoides tanto exógenos como endógenos (20). Una exposición prolongada a corticoides se asocia a déficits en memoria declarativa, obteniéndose también peores resultados en diversas pruebas cognitivas $(20,27)$. Respecto al tiempo necesario de exposición a los corticoides para que tengan lugar los cambios morfológicos, en ratas la atrofia hipocampal aparece sólo después de 3 semanas de intenso estrés. En el hombre, es evidente la alteración cognitiva después de una única dosis de $1 \mathrm{mg}$ de dexametasona o de $10 \mathrm{mg}$ de cortisol. Este efecto no es debido a la neurodegeneración pero parece ser mediado por la acción agonista de los corticosteroides y sus metabolitos en los receptores GABAa. Es realmente imposible estimar cuál es la duración crítica y necesaria de una hiperexposición a corticoides para que ocurran los cambios estructurales en el hipocampo. Respecto a si este tipo de neurodegeneración es reversible, la reducción del volumen hipocampal es visible en pacientes con historia de trastornos afectivos años después del último episodio depresivo e incluso en sujetos con niveles normales de cortisol, por lo que estos hallazgos sugieren que al menos una parte de la neurodegeneración inducida por los corticoides, sería irreversible (20).

\section{Resultados de nuestra revisión de casos.}

Se realiza un resumen de nuestra revisión de casos (tabla 2), así como de la revisión llevada a cabo por parte de Lewis y Smith en 1983 (tabla 3). 
Tabla 2 .

Resumen de nuestra revisión de casos.

\begin{tabular}{|c|c|c|c|c|}
\hline Hipomanía/manía & Sexo & Edad & $\begin{array}{l}\text { Enfermedad } \\
\text { subyacente }\end{array}$ & $\begin{array}{c}\text { Dosis de prednisona o } \\
\text { equivalente }\end{array}$ \\
\hline Benyamin et al 2008 & Varón & 67 & Dolor cervical crónico & $100 \mathrm{mg}$ \\
\hline Mototsugu et al. 2012 & Mujer & 66 & Neoplasia gástrica & $\begin{array}{l}\text { Dexametasona } 6.6 \\
\mathrm{mg} / \text { día }\end{array}$ \\
\hline $\begin{array}{l}\text { Zapata E., Castiella A., } \\
\text { Zubiaurre L. } 2010\end{array}$ & Mujer & 58 & Hepatitis autoinmune & Prednisona $60 \mathrm{mg} /$ día \\
\hline $\begin{array}{l}\text { Milton G. Roxanas et } \\
\text { al. } 2012\end{array}$ & $\begin{array}{l}\text { Hombres y mujeres. } \\
n=20\end{array}$ & & Múltiples patologías & $\begin{array}{l}\text { 100mg/día (dosis } \\
\text { media equivalente de } \\
\text { prednisona } 336 \mathrm{mg} / \text { día) }\end{array}$ \\
\hline Cerullo. 2008 & Hombre & 69 & Linfoma no Hodgkin & $?$ \\
\hline Kenna et al. 2011. & $\begin{array}{l}\text { Hombres y mujeres. } \\
\mathrm{n}=27\end{array}$ & $18-93(44.5+-17.7)$ & Multiples patologías & $15-666,7 \mathrm{mg}$ \\
\hline Kenna et al. 2011 & Mujer & 85 años & Arteritis de la temporal & Prednisona 60mg/día \\
\hline $\begin{array}{l}\text { Kalambokis G, } \\
\text { Konitsiotis S, Pappas } \\
\text { D, Tsianos EV.2006 }\end{array}$ & Varón & 56 & $\begin{array}{l}\text { Enfermedad de } \\
\text { Addison }\end{array}$ & $\begin{array}{l}30 \mathrm{mg} \text { hidrocortinosa } \\
+0,1 \mathrm{mg} \text { de } \\
\text { fludrocortisona/día }\end{array}$ \\
\hline Nuestro caso & Varón & 71 & $\begin{array}{l}\text { Adenocarcinoma } \\
\text { broncopulmonar } \\
\text { en tratamiento con } \\
\text { quimioterapia }\end{array}$ & $\begin{array}{l}26.67 \mathrm{mg} / \mathrm{día} \text { de } \\
\text { prednisona durante } \\
\text { dos días }\end{array}$ \\
\hline $\begin{array}{l}\text { Ayadi N., } \\
\text { Cheikhrouhou MR., } \\
\text { Kechaou M., Bahloul } \\
\text { Z., Jawa A. } 2007\end{array}$ & $\begin{array}{l}\text { Hombres y mujeres: } \\
14\end{array}$ & Edad media: 43,35 años & $\begin{array}{l}7 \text { casos de LES y } \\
7 \text { casos de diversas } \\
\text { patologías }\end{array}$ & $\begin{array}{l}60-100 \mathrm{mg} / \text { día (dosis } \\
\text { media de } 77,14 \mathrm{mg} / \text { día })\end{array}$ \\
\hline $\begin{array}{l}\text { Kimmel RJ., } \\
\text { Combs H. } \\
2012 \\
\end{array}$ & Mujer & 55 años & $\begin{array}{l}\text { Cáncer de mama con } \\
\text { metástasis cerebrales }\end{array}$ & Dexametasona $4 \mathrm{mg} / \mathrm{d}$ \\
\hline $\begin{array}{l}\text { Siddiqui Z., } \\
\text { Ramaswamy S., } \\
\text { Petty F. } \\
2005\end{array}$ & Hombre & 52 años & Transplante hepático & $\begin{array}{l}\text { Metilprednisona } 50 \mathrm{mg} \\
\text { iv/6 horas ( } 4 \text { dosis) y } \\
\text { después, } 40 \mathrm{mg} \text { iv ( } 4 \\
\text { dosis). }\end{array}$ \\
\hline
\end{tabular}

Tabla 3.

Resumen de la revisión de casos de Lewis y Smith (1983).

\begin{tabular}{|lllll|}
\hline \multicolumn{6}{|l|}{ Lewis DA., Smith RE. 1983 Revisión de 14 casos } & & \\
\hline Hombre & 62 años & Transplante renal & Prednisona $29 \mathrm{mg} /$ día (54 días) & Manía \\
\hline Mujer & 61 años & Púrpura Trombótica Idiop & Prednisona $40 \mathrm{mg} /$ día (2 días) & Depresión \\
\hline Mujer & 48 años & Asma & Prednisona $60 \mathrm{mg} /$ día (5 días) & Manía \\
\hline Hombre & 62 años & Enf. Crohn & Prednisona $60 \mathrm{mg} /$ día (8 días) & Manía \\
\hline Mujer & 58 años & EPOC & Prednisona $60 \mathrm{mg} /$ día (4 días) & Delirium \\
\hline Mujer & 47 años & Asma & Prednisona $60 \mathrm{mg} /$ día (4 días) & Manía \\
\hline Hombre & 48 años & Cáncer pulmón & Dexametasona $8 \mathrm{mg} /$ día (7 días) & Manía \\
\hline Mujer & 53 años & Asma & Prednisona $100 \mathrm{mg} /$ día (21 días) & Manía \\
\hline Mujer & 22 años & Lupus Erit. Sist. & Prednisona $70 \mathrm{mg} /$ día (21 días) & Psicosis \\
\hline Mujer & 18 años & Lupus Erit. Sist. & Prednisona $60 \mathrm{mg} /$ día (14 días) & Psicosis \\
\hline Hombre & 53 años & Púrpura Trombótica Idiop & Prednisona $97 \mathrm{mg} /$ día (34 días) & Manía \\
\hline Hombre & 62 años & EPOC & Prednisona $20 \mathrm{mg} /$ día (1 día) & Delirium \\
\hline Hombre & 71 años & Lupus Erit. Sist. & Prednisona $60 \mathrm{mg} /$ día (21 días) & Delirium \\
\hline Hombre & 36 años & Púrpura Trombótica Idiop. & Prednisona $48 \mathrm{mg} /$ día (32 días) & Delirium \\
\hline
\end{tabular}




\section{Caso clínico.}

- Motivo de consulta y antecedentes:

o Motivo de consulta: Desde el servicio de oncología se realiza una interconsulta al servicio de Psiquiatría por agitación psicomotriz.

o Antecedentes personales médicos: no RAMc, no DM, no dislipemia. Hipertensión arterial. Tratamiento habitual: bisoprolol/hidroclorotiazida 10/25mg, omeprazol, alprazolam, ibuprofeno. Desde hace unos meses mantiene seguimiento por Oncología Médica por Adenocarcinoma broncopulmonar con metástasis pleurales (estadío IV), estando en tratamiento a nivel ambulatorio con ciclos de quimioterapia con cisplatino y permetrexed, además de corticoides en las primeras horas tras el ciclo $(4 \mathrm{mg} /$ día de dexametasona, equivalentes a $26.67 \mathrm{mg} /$ día de prednisona). En el momento del ingreso había recibido el cuarto ciclo de quimioterapia.

o Antecedentes personales toxicológicos y psiquiátricos: ex-fumador de 10-15 años/paquete, abstinencia de 30 años de evolución. No otros hábitos tóxicos. Personalidad premórbida con tendencia a la introversión. Su familia lo describe con el apelativo de "El callao".

o Antecedentes biográficos: Casado, con 3 hijos. Vive con su esposa. Buenas relaciones familiares. Actividad laboral en la industria del calzado, jubilado.

o Antecedentes familiares: múltiples antecedentes familiares de tipo oncológico (madre LLC; hermana con neoplasia uterina; hijo con linfoma cutáneo). Padre con demencia vascular. Sin antecedentes familiares psiquiátricos de interés.

- Enfermedad actual y exploración psicopatológica:

o Enfermedad actual: hombre de 71 años de edad que ingresa en Oncología Médica por alteraciones conductuales en el domicilio de dos semanas de evolución, llegando a precisar ingreso por dificultades para la contención ambulatoria. Realizan IC al servicio de Psiquiatría para valoración y ajuste farmacológico. En la entrevista inicial mantenida con los familiares destacan: verborrea, agitación psicomotriz, desorientación, temblor y alucinaciones de intensidad creciente, con empeoramiento en los últimos cuatro días previos al ingreso en los que aparecieron insomnio, discurso verbal y escrito incoherente, tics verbales y posturales, irritabilidad y verbalizaciones de ideas de contenido místico y megalomaníaco. En los días iniciales del ingreso se había pautado tratamiento con haloperidol $4 \mathrm{mg} /$ día, lorazepam $1 \mathrm{mg} / 12 \mathrm{~h}$, haloperidol $1 / 2$ ampolla de rescate, tiaprida y midazolam, sin mejoría de la clínica. Los familiares recordaban alteraciones conductuales en relación con ciclos previos de quimioterapia: en los primeros 3-4 días tras la administración del tratamiento el paciente se mostraba hipertímico, logorreico, con aumento de 
energía y realización de diferentes actividades. Posteriormente, durante 10 días, había un cambio en la clínica predominando el ánimo triste, la apatía, la astenia y la tendencia a la clinofilia. Pasados estos días, normalización afectiva y conductual, con retorno a su estado habitual.

o Exploración psicopatológica al ingreso: el paciente estaba consciente e hipoproséxico, no siendo posible valorar orientación. Ecopraxias y temblor leve. Discurso incoherente, con tendencia a la tangencialidad, pararrespuestas, ecolalia y disfemia, del que podrían desprenderse ideas de contenido místico. Probables alucinaciones visuales.Labilidad emocional marcada y afecto incongruente, sin signos de ansiedad. Empeoramiento vespertino-nocturno. Como primera hipótesis diagnóstica nos planteamos un cuadro confusional, procediendo a la ampliación del estudio orgánico y al ajuste farmacológico, introduciéndose haloperidol gotas: 15-15-30 y una dosis extra de rescate.

- Pruebas complementarias:

o Análisis de sangre: hemograma, bioquímica incluyendo perfil ferrocinético y tiroideo, ácido fólico y vitamina B12, dentro de parámetros normales. VSG $86 \mathrm{~mm}$.

o Serología VHB, VHC, VIH, Sífilis: negativo.

o Análisis de orina: sin alteraciones.

o Punción lumbar: GRAM y cultivo negativos.

o RMN cerebral: sin hallazgas significativos.

o Electroencefalograma: resultados dentro de la normalidad.

- Diagnóstico diferencial:

o Metástasis a nivel cerebral o en líquido cefalorraquídeo: diagnóstico que quedó descartado con el estudio orgánico.

o Delirium: si bien en la primera valoración predominaba la clínica confusional, en las siguientes 24-48 horas la clínica afectiva pasó a un primer plano.

o Manía primaria vs secundaria.Aunque podríamos plantearnos la posibilidad de una manía primaria, sabemos que su debut en un paciente de más de 35 años de edad, sin antecedentes personales ni familiares de trastornos del estado de ánimo y coincidiendo con las primeras sesiones de tratamiento con quimioterápicos y corticoides la hacen altamente improbable. Además son síntomas característicos de una manía secundaria el deterioro cognitivo moderado, que en nuestro paciente fue un delirium franco los primeros días, la labilidad anímica, las alucinaciones visuales y los delirios (28).

» Manía secundaria a cisplatino: nos planteamos como posibilidad diagnóstica un secundarismo debido al cisplatino. Esta opción fue descartada rápidamente por el servicio de oncología responsable del paciente, al no hallar correlatos en la literatura. Mototsugu et al (13) publicaron el caso de una mujer de 66 años con síntomas maníacos, planteando como hipótesis diag- 
nóstica que el cuadro pudiera deberse al tratamiento antineoplásico con cisplatino; no obstante, rechazaron esta hipótesis al no hallar referencias bibliográficas similares o relacionadas con su caso, que se trataba de un cáncer gástrico metastásico.

Manía secundaria a corticoides: el diagnóstico de los trastornos psiquiátricos inducidos por corticoides es un diagnóstico de exclusión. En pacientes geriátricos, como nuestro caso, deben descartarse una gran variedad de patologías médicas que pueden dar síntomas similares a la psicosis, el delirium, la manía y la depresión. Entre las entidades a descartar se encuentran $(1,10)$ : trastornos psiquiátricos primarios (descartado en nuestro paciente dada la ausencia de antecedentes personales psiquiátricos); encefalopatías tóxicas, metabólicas e infecciosas (se planteó como posibilidad diagnóstica la presencia de metástasis a nivel cerebral o espinal, por lo que se realizó una punción lumbar, una RMN cerebral, EEG y diversos análisis que arrojaron resultados normales); delirium (presente en los primeros días del ingreso); accidentes cerebrovasculares (la clínica no lo sugería, y las pruebas complementarias eran normales); otros trastornos neuropsiquiátricos (como Parkinson, la clínica no lo sugería y la evolución tampoco); traumatismos (no lo refería ni el paciente, ni la familia ni el servicio de oncología) y neoplasias (descartados con las pruebas de imagen).

o Reacción vivencial anormal de características maníacas. Frente a las reacciones vivenciales que Jaspers (29) delimitó en base a tres criterios principales (que la situación reactiva no hubiera surgido a no ser por la vivencia que la originó, que su contenido se halla en relación comprensible con respecto a su causa y que la situación es temporalmente dependiente de su causa y, por tanto, cesará cuando ésta desaparezca), las reacciones vivenciales anormales se caracterizan por su alejamiento del promedio de las normales a causa de su extraordinaria intensidad, de su falta de adecuación respecto al motivo que las provoca o bien por la anormalidad de su duración. En general, las reacciones a vivencias exteriores (como puede ser una enfermedad grave) son parcialmente independientes del carácter individual. Estas reacciones son descritas de acuerdo con los cuadros clínicos que más llamativos resultan al exterior (30). En el caso concreto de las manías reactivas podemos decir que son cuadros que suelen carecer de importancia clínica pues no suelen alcanzar proporciones anormales. Aunque nuestro paciente presentó una negación de la grave enfermedad de su hijo (linfoma cutáneo) en años previos y podamos interpretar la reacción maníaca actual como un mecanismo de defensa, en el sentido descrito por Klein (31), ante una situación vital altamente estresante y difícilmente asumible por el individuo, lo cierto es que la intensidad de los síntomas maníacos desarrollados, que llegan a acompañarse de síntomas psi- 
cóticos, lo hacen poco plausible. Además la relación temporal entre el inicio de la enfermedad (varios meses antes) y la aparición del cuadro maníaco no es clara. Siendo más estrecha la correlación entre los episodios de hipomanía que presentó en los tres ciclos previos de quimioterapia y el episodio maníaco descrito tras el último tratamiento quimioterápico.

- Juicio clínico: una vez descartadas otras posibilidades etiológicas y diagnósticas, consideramos la clínica como un episodio maníaco inducido por corticoides (Trastorno del estado de ánimo inducido por sustancias, con síntomas maníacos).

- Tratamiento y evolución: en los días siguientes el paciente fue valorado regularmente pasando de un estado de delirium a un episodio de características maniformes; durante la evolución presentó episodios de agitación, desinhibición sexual, ideación delirante de corte místico-religioso y megalomaníaco, dificultad para mantener límites interpersonales, asociaciones laxas e interpretaciones de corte delirante puntuales. Se retiró el tratamiento previo, que no había sido efectivo, y se introdujo tratamiento con quetiapina a dosis máximas de $150 \mathrm{mg} /$ día (25mg-25mg-100mg) y clonazepam $3 \mathrm{mg} /$ día $(0.5 \mathrm{mg}-0.5 \mathrm{mg}-2 \mathrm{mg})$. Decidimos usar quetiapina ante el fracaso de los neurolépticos clásicos utilizados, sus propiedades antimaníacas, eutimizantes y sedantes, así como su buena tolerabilidad y escasos efectos adversos en pacientes con patología médica comórbida, como nuestro paciente. Para valorar la eficacia del tratamiento, además de la exploración psicopatológica, decidimos utilizar la escala de Young por ser un instrumento sencillo, sensible y efectivo para la valoración de los síntomas maníacos. Es una escala que tiene 11 ítems (ánimo elevado, psicomotricidad, interés sexual, sueño, irritabilidad, lenguaje, contenido del pensamiento, agresividad, aspecto físico e insight) y que había sido usada en otros casos similares al nuestro (7). Al ingreso, la puntuación de Young era de 31 puntos; a los seis días de ingreso, 33 puntos; al alta 2 puntos. Como se puede ver, progresivamente la sintomatología maníaca remitió; al alta el paciente estaba eutímico y realizaba crítica de la clínica. Decidimos mantener el tratamiento con clonazepam 3mg/ día $(0.5-0.5-2 \mathrm{mg})$ y quetiapina $125 \mathrm{mg} /$ día $(25-0-100 \mathrm{mg})$, con pauta descendente según evolución. En las sucesivas revisiones mantenidas con el servicio de Oncología el paciente estaba asintomático psicopatológicamente. 
10. Prevención, vigilancia y tratamiento de las manías secundarias a terapia con corticoesteroides.

- Tratamiento:

Según las fuentes consultadas, el tratamiento de primera línea de la manía secundaria a corticoides es la reducción de la dosis del corticoide o la interrupción del mismo; cuando esto no es posible o la sintomatología es severa puede ser necesario el uso de fármacos adyuvantes $(1,3,6,8,12-13)$. Entre estos se incluyen antipsicóticos típicos (si bien se han relacionado con alteraciones en el hipocampo, sumado al riesgo de síntomas extrapiramidales) (5) y atípicos (sobre todo olanzapina, risperidona y quetiapina), litio (que incluso se ha usado profilácticamente con buenos resultados) $(7,32)$, carbamazepina, ácido valproico, clonazepam, lamotrigina, gabapentina, fenitoína y TEC, según la gravedad de la clínica $(1,7-8,10,13$, 27). Parece que a nivel farmacológico los neurolépticos atípicos son los fármacos de primera línea, siendo el litio un fármaco de segundo nivel.

o Antipsicóticos de segunda generación:

La olanzapina ha demostrado ser un fármaco eficaz y bien tolerado en el tratamiento de los trastornos del estado de ánimo inducidos por corticoides. En 2004 el grupo de Brown publicó un estudio en el Journal of Affective Disorders (32) en el que se incluyeron 12 pacientes de entre 18 y 65 años de edad, sin antecedentes psiquiátricos personales, que presentaron síntomas maníacos o mixtos (definidos como una puntuación mayor o igual a 10 en la escala de Young) en relación a un tratamiento previo con corticoides. Además de la escala de Young utilizaron escalas como la Hamilton y otras para medir los secundarismos del neuroléptico (Simpson Angus Extrapyramidal Rating Scale, Abnormal Involuntary Movement Scale, Barnes Akathisia Scale). A los pacientes se les administró inicialmente $2.5 \mathrm{mg}$ de olanzapina, dosis que se fue aumentando de forma progresiva (aumentos de 2.5$5 \mathrm{mg}$ ) semanalmente según la respuesta y los efectos secundarios. El tratamiento con olanzapina se mantuvo durante cinco semanas, mostrando los resultados una mejora importante en la escala de Young (pasando de 15.25 de media inicial a 5.83), sin cambios significativos en las escalas que medían los efectos adversos. Se midieron también los niveles de glucosa en sangre al principio y al final del estudio, siendo la elevación no significativa. Concluyen, por tanto, que la olanzapina es un fármaco eficaz y bien tolerado en este tipo de pacientes (32).

Las dosis utilizadas de risperidona oscilan entre 1 y $4 \mathrm{mg}$ diarios, con tiempos de mejora de días-semanas. Se ha utilizado en gran variedad de síntomas, sobre todo en hipomanía y alucinaciones.

Respecto a la quetiapina, está publicado el caso de un varón de 52 años que desarrolló síntomas maníacos tras el tratamiento con corticoides. Inicialmente presentaba 31 puntos en la escala de Young; se inició tratamiento con quetiapina $25 \mathrm{mg}$ 
en dosis única nocturna, asociando $12.5 \mathrm{mg} / 12 \mathrm{~h}$ de rescate. En las primeras horas del tratamiento se evidenció una clara mejoría clínica; pasados 5 días la puntuación en la escala de Young era de 5 puntos. Se decidió introducir quetiapina por sus propiedades antimaníacas y eutimizantes, su poder sedativo y su vida media corta, siendo útil en pacientes complejos con patología médica asociada, pudiendo considerarse un fármaco útil como tratamiento profiláctico de la manía corticoidea (33).

Respecto al uso de aripiprazol hemos consultado el caso clínico de una mujer de 55 años con cáncer de mama y metástasis cerebrales que había recibido tratamiento con dexametasona $4 \mathrm{mg}$ y que presentó agitación, insomnio, irritabilidad, verborrea, ánimo expansivo e ideas delirantes de perjuicio. Se introdujo tratamiento con aripiprazol $10 \mathrm{mg} /$ día y clonazepan $0.5 \mathrm{mg} /$ día en toma nocturna. Tras cinco días de tratamiento se normalizó la clínica. La paciente estuvo en tratamiento con aripiprazol durante cinco semanas; tras su retirada, no volvió a presentar síntomas psiquiátricos. Este caso pone de manifiesto que el aripiprazol constituye un fármaco eficaz para el tratamiento de la psicosis y la manía inducida por corticoides (26).

o Eutimizantes/antiepilépticos:

El ácido valproico es un eutimizante caracterizado por su amplio margen de seguridad, es fácilmente medible en sangre y causa menos efectos secundarios neurológicos en comparación con otros estabilizadores. Presenta la ventaja frente a carbamazepina que no induce el metabolismo glucocorticoideo, lo que lo hace un fármaco útil y seguro en el tratamiento de la manía esteroidea (7), tal y como concluye Roxanas en un estudio realizado con 20 pacientes con síntomas maniformes derivados del tratamiento con corticoides a los que se administró valproato como tratamiento. Este estudio incluye 20 pacientes ( 8 hombres y 12 mujeres), con una media de edad de 53 años, que fueron tratados con valproato. Ninguno de ellos tenía antecedentes psiquiátricos. La dosis media de corticoides fue de $100 \mathrm{mg} /$ día (dosis media equivalente de prednisona $336 \mathrm{mg} /$ día), y la de valproato $500 \mathrm{mg}$ dos veces al día. El primer día la media en la escala de Young era de 25 puntos, siendo al tercer día de 2,5. Concluyen, por tanto, que el valproato produce un descenso significativo en la escala de Young, mejorando los síntomas maníacos, y sugieren que podría ser un buen tratamiento profiláctico de la manía inducida por corticoides (7).

La carbamacepina y gabapentina pueden ser útiles en la prevención de la aparición de cuadros maníacos en pacientes en tratamiento con dosis repetidas de corticoides (34-35).

Parece que la lamotrigina atenúa los efectos atróficos de los corticoides sobre la amígdala, pero no queda tan claro su efecto a nivel sintomático, al no evidenciarse cambios en la escala de Young al ser comparada con placebo (36).

El levetiracetam no parece ser efectivo en el tratamiento de los síntomas depresivos y/o maníacos secundarios a corticoides (37). 
Respecto a otros fármacos es posible que en el futuro se abra el abanico de posibles tratamientos, por ejemplo que actúen específicamente sobre los receptores GR o de CRH o que interaccionen con los sistemas serotoninérgico, gabaérgico o glutamatérgico, con el objetivo de prevenir los efectos neurotóxicos de los corticoides (20).

- Prevención.

A nivel preventivo es importante que el clínico informe a paciente y familiares sobre el riesgo de desarrollar síntomas psiquiátricos al iniciar un tratamiento con corticoides $(1,25)$, así como mantener una supervisión estrecha durante las primeras semanas del tratamiento $(1,16)$ con el objetivo de detectar precozmente la sintomatología.

A nivel farmacológico, parece que el litio presenta eficacia terapéutica como tratamiento preventivo $(11,38,39)$; la quetiapina, el ácido valproico, la carbamazepina y la gabapentina también serían fármacos a considerar $(7,12,33-35)$. Pese a que no existen guías que indiquen cuando iniciar un tratamiento preventivo, se consideran candidatos al mismo aquellos pacientes con historia personal de complicaciones psiquiátricas secundarias a corticoides y en aquellos en los que la aparición de síntomas psiquiátricos suponga un grave riesgo (12).

\section{Conflicto de intereses.}

Los autores declaran no tener conflictos de intereses.

\section{Bibliografía.}

(1) Kenna HA, Poon AW, de los Angeles P, Koran LM. Psychiatric complications of treatment with corticosteroids: review with case report. Psychiatr Clin Neurosci 2011; 65: 549-560.

(2) Clark LD, Bauer WD, Cobb S. Preliminary observations on mental disturbances occurring in patients under therapy with cortisone and ACTH. New Engl J Med 1952; 246: 205-216.

(3) Ayadi N, Cheikhrouhou MR, Kechaou M, Bahloul Z, Jawa A. Hypomanie sous corticotherapie. À propos de 50 cas. Therapie 2007; 5:443-447.

(4) Brown ES. An Epidemiological Approach to "Steroid Psychosis". Am J Psychiatry 2012; 169(5):447-449.

(5) Téllez-Vargas, J. Trastornos neuropsiquiátricos inducidos por la terapia con corticoides. http://www.presenciaactiva.com/contingut/m_pujadocuments/documents/File/Trastornos_neuropsiquiatricos_inducidos_por_la_terapia_con_corticoides.pdf.

(6) Zapata E, Castiella A, Zubiaurre L. Corticosteroid-induced mania in a patient with autoimmune hepatitis. European Journal of Gastroenterology \& Hepatology 2010; 22: 500-501. 
(7) Roxanas MG., MB BS., FRANZCP., Hunt GE., Ph D. Rapid reversal of corticosteroid-induced mania with sodium valproate: a case series of 20 patients. Psychosomatics 2012; 53: $575-581$.

(8) Muzyk AJ, Holt S, Gagliardi JP. Corticosteroid psychosis: stop therapy or add psychotropics? Current Psychiatry 2010; vol. 9, $\mathrm{n}^{\circ} 1$.

(9) Fardet L, Petersen I, Nazareth I. Suicidal behavior and severe neuropsychiatric disorders following glucocorticoid therapy in primary care. Am J Psychiatry 2012, AiA: 1-7.

(10) Cerullo MA. Expect psychiatric side effects from corticosteroid use in the elderly. Geriatrics January 2008; volume 63, number 1.

(11) Lewis DA, Smith RE. Steroid-induced psychiatric syndromes. A report of 14 cases and a review of the literature. J Affect Disord 1983;5:319-32.

(12) Cerrullo MA. Corticosteroid-induced mania: Prepare for the unpredictable. Current Psychiatry 2006; 5(6): 43-50.

(13) Mototsugu Matsunaga et al. Hypomaniac episode during recurrent gastric cancer treatment: report of a rare case and literature review. Jpn J Clin Oncol 2012; 42(10): 961-964.

(14) Organización Mundial de la Salud. Clasificación de los Trastornos Mentales y del comportamiento $10^{\text {a }}$ edición. Madrid: Panamericana. 2000.

(15) American Psychiatric Association. Diagnostic and Statistical manual of mental disorders DSM-IV-TR. Washington: American Psychiatric Association. 2000.

(16) Cornic F, Rousset I. Troubles neuropsychiatriques liés aux corticoids. Rev du Practicien 2008; 58:469-475.

(17) The Boston Collaborative Drug Surveillance Programm. Acute adverse reactions to prednisone in relation to dosage. Clin Pharmacol Ther 1972; 13: 694-698.

(18) Wada K, Yamada N, Sato T, et al. Corticosteroid-induced psychotic and mood disorders: diagnosis defined by DSM IV and clinical pictures. Psychosomatics 2001; 42: 461-466.

(19) Stiefel FC, Breibart WS, Holland JC. Corticosteroids in cancer: neuropsychiatric complications. Cancer Invest 1989; 7: 479-491.

(20) Hoschl C, Hajek T. Hippocampal damage mediated by corticosteroids- a neuropsychiatric research challenge. Eur Arch Psychiatry Cli Neurosci 2001; 251: Suppl.2:II81-88.

(21) Nishimura K, Harigai M, Omori M, Sato E, Hara M. Blood-brain barrier damage as a risk factor for corticosteroid-induced psychiatric disorders in systemic lupus erythematosus. Psychoneuroendocrinology 2008; 33: 395-403.

(22) Sirois F. Steroid psychosis: a review. Gen Hosp Psychiatry. 2003; 25: 27-33.

(23) Bolanos SH, Kahn DA, Hanczyc M, Bauer MS, Dhanavi N, Brown ES. Assesment of mood status in patients receiving long-term corticosteroid therapy and in controls with patient-rated and clinician-rated scales. Ann Allergy Asthma Immunol 2004; 92: 500-505.

(24) Brown ES, Woolston DJ, Frol A, Bobadilla L, Khan DA, Hanczyc M et al. Hippocampal volume, spectroscopy, cognition and mood in patients receiving corticosteroid therapy. Biol Psychiatry 2004; 55: 538-545.

(25) Patten SB, Neutel CI. Corticosteroid-induced adverse psychiatric effects. Drug Safety 2000; 22: 111-122.

(26) Kimmel RJ, Combs H. Steroid-Induced Mania Treated with Aripiprazole. Psychosomatics 2012;53:181-183. 
(27) Brown E.S. Effects of glucocorticoids on mood, memory and the hippocampus. Ann. N.Y. Acad. Sci 2009; 1179: 41-55.

(28) Mc Daniel JS, Johnson KM, Rundell JR. Mania. En Rundell JR, Wise MG. editors. Fundamentos de la psiquiatría de enlace. Barcelona: Psiquiatría editores SL, 2002; p.179-198.

(29) Jaspers K. Psicopatología general. $2^{\mathrm{a}}$ ed. Mexico: Fondo de cultura económica, 1993.

(30) Schneider K. Patopsicología clínica. $4^{a}$ ed. Madrid: Paz Montalvo,1975.

(31) Laplanche J, Pontalis J-B. Diccionario de psicoanálisis. $3^{\mathrm{a}}$ ed. Barcelona: Labor, 1983.

(32) Brown E.S, Chamberlain W, Dhanani N, Paranjpe P, Carmody T.J, Sargeant M. An open-label trial of olanzapine for corticosteroid-induced mood symptoms. Journal of Affective Disorders 2004; 84: 277-281.

(33) Siddiqui Z, Ramaswamy S, Petty F. Quetiapine therapy for corticosteroid-induced mania. Can J Psychiatry 2005;50:77-8.

(34) Wada K, Yamada N, Yamauchi Y, Kuroda S. Carbamazepine treatment of corticosteroid-induced mood disorder. J Affect Dissord 2001; 65: 315-317.

(35) Ginsberg DL, Sussman N. Gabapentin as prophylaxis against steroid-induced mania. Can J Psychiatry 2001;46: 455-456.

(36) Desai S, Khanami S, Shad MU, Brown ES. Attenuation of amígdala atrophy with lamotrigine in patients receiving corticosteroid therapy. J Clin Psychopharmacology 2009; 29: 284-287.

(37) Brown ES, Frol AB, Khan DA, Larkin GL, Bret ME. Impact of levetiracetam on mood and cognition during prednisone therapy. Eur Psychiatry 2007; 22: 448-452.

(38) Falk WE, Mahnke MW, Poskanzer DC. Lithium prophylaxis of corticotrophin-induced psychosis. JAMA 1979; 241: 1011-1012.

(39) Brown ES. Management of psychiatric side effects associated with corticosteroid. Expert Rev Neurother 2003; 3: 69-75. 